\title{
Effect of coolant temperature and cooling time on fractional crystallization of biodiesel and glycerol
}

\author{
Shafirah Samsuri", Nurul Aini Amran, Loh Jia Zheng, Muhammad Muhaimin Mohd Bakri \\ Chemical Engineering Department, Universiti Teknologi PETRONAS, 32610 Seri Iskandar, Perak, Malaysia \\ *Corresponding author: shafirah.samsuri@utp.edu.my
}

\section{Article history}

Received 19 November 2017

Accepted 10 December 2017

\begin{abstract}
In the middle of the era of technology and fast-growing industry nowadays, biodiesel (methyl ester) has been identified as a sustainable fuel to replace petroleum. Hence, the separation and purification of the methyl ester after the trans-esterification process is essential since the purification of methyl ester is compulsory for the fuel industry in order to fulfill the strict global standard particulars for methyl ester. One of the current method used for separation and purification of methyl ester is called wet washing technology. However, this technology has its own drawbacks such as huge amount of water consumption as well as high cost for the wastewater treatment process. Due to these drawbacks, fractional crystallization process is proposed in order to save water and minimize the time consumed for the process of separation and purification of methyl ester. Fractional crystallization is a process that involved a solid-liquid separation where the process takes place in a crystallizer. In this process, methyl ester was separated from the glycerol based on their differences in term of melting point of the components. By observing the layer formation of the components, the effect of cooling time and coolant temperature on the performance of separation and purification of methyl ester by fractional crystallization process were studied. The purified methyl ester obtained was placed in a gas chromatographer in order to test the purity of methyl ester and to evaluate the efficiency of the process based on two parameters that has been investigated which are effective partition constant $(\mathrm{K})$ and concentration efficiency (Eff). It was found that to achieve highest effectiveness of fractional crystallization system and highest concentration efficiency where $\mathrm{K}$ and Eff are 0.51 and $47.71 \%$, respectively, the fractional crystallization must operate at coolant temperature range of -10 to $-12^{\circ} \mathrm{C}$ and cooling time range of 30 to 35 minutes.
\end{abstract}

Keywords: Fractional crystallization, separation, purification, methyl ester, glycerol

(C) 2017 Penerbit UTM Press. All rights reserved

\section{INTRODUCTION}

Biodiesel (methyl ester) has become an alternative fuel of diesel and it has been greatly increasing due to its characteristics, biodegradability and manageability. Berrios and Skelton (2008) stated that the process of separation and purification of methyl ester is obligatory in order to fulfill the strict global standard for methyl ester. The conventional process was done to remove an alcohol via various methods such as wet or dry washing as well as vacuum distillation in order to remove catalyst, soap and triglycerides (Berrios and Skelton, 2008). Therefore, those methods are not fully recommended because of its drawback that can lead to a negative contribution such as environmental disposal problems (Atadashi et al., 2011). In addition, it consumed a huge amount of water that can lead to huge energy consumptions as well as consumed large capital cost of wastewater treatments. Urich and Jones (2006) define crystallization as a separation process that yields a solid production from a solution or a melt. The main feature distinguishing crystallization from other thermal separation processes is the fact that it leads to a solid production. Due to the problems of conventional purification by wet washing technology, crystallization process has shown its capability to produce a very high purity product from solutions that contained significant amount of impurities with a minimal energy input compared to other unit operations (Ulrich and Jones, 2006).

Freeze concentration has been started with a new method of concentration of impurities from organic compound by progressive freezing (Matthews and Coggeshall, 1959), where crystallization by freezing, fractional melting, or zone melting techniques are the methods that have come forward in obtaining ultra-high of the products. Many researchers have shown the advantages of freezing in purification of mixtures by separating the two components. Freezing is one of the application of crystallization that has been successfully applied to the management of wastewater, liquid food concentration, food juice concentration and also sea water desalination (Aider and Ounis, 2012; Lemmer et al., 2001; Mandri et al., 2011; Rane and Uphade, 2016; Rich et al., 2012).

For separation and purification of methyl ester, there are several alternative physical steps that can be applied such as freezing, subsequent removal and melting. When fractional crystallization takes place in purifying methyl ester, the glycerol was separated by forming a solid phase while the methyl ester was remained in liquid phase. The separation between solid and liquid phase is relatively easy. The liquid is allowed to drain out of the cold surface (Auleda et al., 2011). It does not require wash columns, centrifuges or presses. Hence, this 
process can be considered as a cost-effective and environmental friendly process since there is reduction of operating units.

In crystallization process, crystallization from solution and crystallization from melts are often used (Samsuri et al., 2016) Crystallization from solution is a homogeneous mixture of more than one species. Crystallization from melt most correctly refers to a pure molten solid, such as molten silicon. Melt crystallization is a technique used for purification and separation of mixtures of chemicals and metals. The fraction of the crystallizing component in melt crystallization is commonly present in higher amount than all the other mixture components together (Fukui et al., 2013). The advantages of melt crystallization are in the relatively low energy demand of the freezing process and in the high selectivity of crystallization. In such processes a multiple of the heat of crystallization is needed, which reduces the energy benefits of melt crystallization compared to distillation (Wintermantel and Wellinghoff, 2001). Fractional crystallization is one of the melt crystallization, a process by which a chemical compound is separated into its component. Thus, the aim of this study is to investigate the effect of coolant temperature and cooling time of fractional crystallization on separation and purification of methyl ester and glycerol. The experiment was executed by varying the coolant temperature and cooling time.

\section{EXPERIMENTAL}

\section{Materials}

Methyl ester and glycerol were used in fractional crystallization experiments while ethylene glycol solution of $50 \%(\mathrm{v} / \mathrm{v})$ with water was used for the coolant. Only glycerol is being selected to be used as a contaminant because glycerol is a major contaminant compared to others such as catalyst, soap and triglycerides.

\section{Methods}

Fig. 1 shows the setup apparatus for fractional crystallization system. A cylindrical crystallizer was made from stainless steel that has a characteristic of high resistance towards steel corrosion by some of the composition in the solution. The crystallizer was equipped with a stirrer in order to improve the mechanism of separation as the movement of the methyl ester at solid-liquid was promoted. In the beginning of the experiment, glycerol and methyl ester were mixed in $1: 1$ volume ratio in order to obtain crude methyl ester. Then, the crude methyl ester was fed into the crystallizer and was immersed in the water bath at the desired temperature. The stirrer was turned on to stir the crude methyl ester and it was left in the crystallizer until all the glycerol was separated from methyl ester. The crystallizer was taken out from the water bath after stirring process was stopped at the desired cooling time. The pure methyl ester was drained out from the crystallizer and the volume of pure methyl ester was measured. The solid phase of glycerol was detached from the cooled surface and left at room temperature for it to melt. Every sample taken was analyzed using gas chromatographer for the purity measurement.

To study the effect of coolant temperature on effective partition constant $(\mathrm{K})$ and concentration efficiency (Eff), stirring rate and coolant time for fractional crystallization process were kept constant at $300 \mathrm{rpm}$ and 30 minutes, respectively. Six sets of experiments were conducted from $-6^{\circ} \mathrm{C}$ to $-16^{\circ} \mathrm{C}$ with the decreasing of coolant temperature by $-2^{\circ} \mathrm{C}$. For the effect of cooling time on $\mathrm{K}$ and Eff, stirring rate and coolant temperature for fractional crystallization process were kept constant at $300 \mathrm{rpm}$ and $-10^{\circ} \mathrm{C}$, respectively. Five sets of experiments were conducted started from 20 minutes to 40 minutes with an interval of 5 minutes.

\section{Gas analysis chromatography}

The solid and liquid samples were analysed using Calrus $600 \mathrm{C}$ Gas Chromatography Mass Spectrometry (GCMS), fitted with a Elite$5 \mathrm{MS}$ column $(30 \mathrm{~mm} \times 0.25 \mathrm{~mm} \times 0.25 \mathrm{um})$. The purpose of using GCMS is to measure the concentration of glycerol in the solid and concentration of methyl ester in the liquid. Helium gas was used as carrier gas while n-heptane was used as diluent. The carrier gas flow is $0.2 \mathrm{~mL} / \mathrm{min}$ and the split ratio is $50: 1$. The injection volume for one run is $0.1 \mathrm{uL}$ and the total run time is 36.5 minutes. First, $35^{\circ} \mathrm{C}$ is hold for 10 minutes. Then, the temperature is rise $10^{\circ} \mathrm{C} / \mathrm{min}$ to $200^{\circ} \mathrm{C}$ and it hold for 10 minutes.

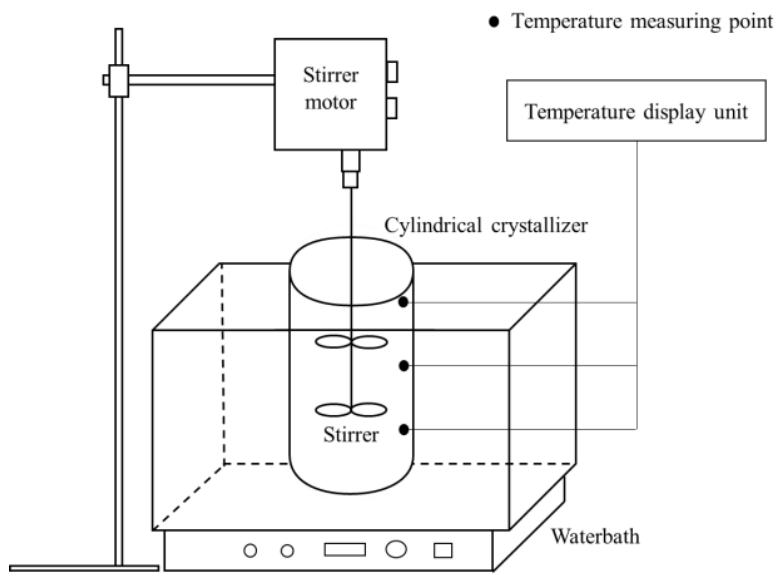

Fig. 1 Apparatus setup for fractional crystallization.

\section{EVALUATION OF SYSTEM EFFICIENCY}

\section{Effective partition constant $(\mathrm{K})$}

For the determinant parameters of the experiment, the effective partition constant $(\mathrm{K})$ act as the most important variable among the other variables in the operation of freeze crystallization system. The value of $\mathrm{K}$ changes between 0 which indicates a complete crystallization and 1 for no crystallization present where the range of $\mathrm{K}$ is between 0 and 1 (Miyawaki et al., 2005). $\mathrm{K}$ between solid and liquid phase is defined in Eq. (1):

$$
K=I-\frac{\log \frac{C_{O}}{C_{L}}}{\log \frac{V_{L}}{V_{O}}}
$$

Where $\mathrm{V}_{\mathrm{L}}$ and $\mathrm{V}_{\mathrm{O}}$ are volume $(\mathrm{mL})$ of methyl ester and initial solution and $C_{L}$ and $C_{O}$ are concentration of methyl ester in the concentrated and initial solution, respectively. The concentration value for each solution was measured by GCMS.

\section{Concentration efficiency (Eff)}

Hernández et al. (2010) stated that the concentration efficiency (Eff) indicates the relationship between the increasing in the concentration of the solution in liquid phase correspond to the concentration of the solid phase as given in Eq. (2):

$$
\text { Eff }=\frac{C_{L}-C_{O}}{C_{L}} \times 100 \%
$$

Where Eff is the concentration efficiency $(\%), \mathrm{C}_{\mathrm{L}}$ is the concentration of methyl ester in liquid phase after freezing process and $\mathrm{C}_{\mathrm{O}}$ is the concentration of the solid phase (Hernández et al., 2010).

\section{RESULTS AND DISCUSSION}

\section{Effect of coolant temperature}

From Fig. 2, the value of $\mathrm{K}$ started to decrease until coolant temperature reached $-10^{\circ} \mathrm{C}$ which having the lowest value of $\mathrm{K}=0.51$. The coolant temperature of $-10^{\circ} \mathrm{C}$ was considered as an ideal temperature that makes the methyl ester accumulates in the solution without entering the solid while the glycerol attracted to the wall and formed a solid. Thus, the concentration of methyl ester in the solution increased. By having a lower coolant temperature, the solid growth rate tend to be higher which caused a higher methyl ester inclusion into the glycerol solid. Therefore, too low of coolant temperature can affect and reduce the purity of methyl ester produced (Caretta et al., 2006). When there is a larger difference in temperature between the 
entering solution and the crystallizer wall, hence it will leads to a higher growth rate of solid formed. In addition, a part of methyl ester might be entrapped within the glycerol solid formed if the solid growth rate is faster than the solute outward movement. In other words, the solute inclusion in the solid formed happens due to the failure of the solute outward movement in order to overtake the growth rate of solid formed (Miyawaki et al., 2016). To overcome this problem, intermediate coolant temperature at $-10^{\circ} \mathrm{C}$ was chosen to make sure that the pure methyl ester can accumulates in the solution without entering the solid formed. When there was less inclusion of methyl ester into the glycerol, a higher amount of pure methyl ester can be obtained.

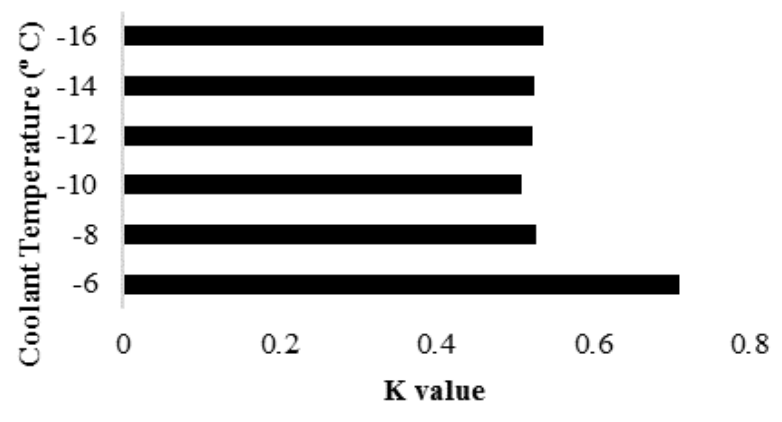

Fig. 2 Graph of $\mathrm{K}$ value for the effect of coolant temperature.

Fig. 3 shows a graph of concentration efficiency for the effect of coolant temperature. From this figure, the value of efficiency increases from $-6^{\circ} \mathrm{C}$ to $-12^{\circ} \mathrm{C}$ and started to decrease slightly until $16^{\circ} \mathrm{C}$. It shows that the solid was able to grow in more ordered pattern and leave the pure methyl ester to accumulate in the solution when the rate of heat transfer is slower at higher coolant temperature. Moreover, the solid formed and pure methyl ester obtained were influenced by the cooling rate in which it may affect the occlusion level. Therefore, the coolant temperature of $-12^{\circ} \mathrm{C}$ has the highest concentration efficiency of $47.71 \%$ compared to the other coolant temperature throughout this experiment.

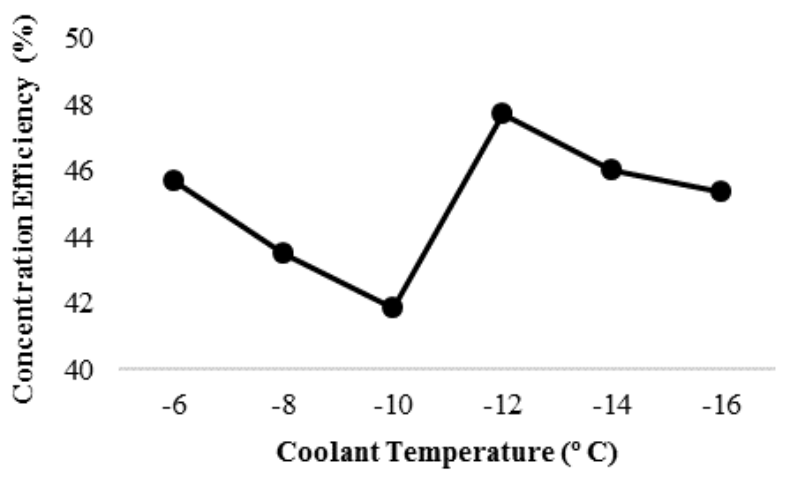

Fig. 3 Graph of concentration efficiency for the effect of coolant temperature.

\section{Effect of cooling time}

Fig. 4 shows a graph of $\mathrm{K}$ value for the effect of cooling time. From this figure, it can be observed that the value of $\mathrm{K}$ decreases until cooling time at 35 minutes and increases slightly after cooling time at 40 minutes. As the cooling time increases, the value of $\mathrm{K}$ decreases which indicates the fractional crystallization has progressed satisfactorily. The concentration of methyl ester in the solution increased as the solid formed increased. Most of the methyl ester had been recovered from the initial solution as a consequence of this process. Solid grows at a faster rate initially and decreases over time due to increased heat transfer resistance and the concentration of methyl ester interface, which then lowers the freezing point, making it more difficult to solidify (Samsuri et al., 2015). This situation will decrease the solid growth rate and making less inclusion of methyl ester into the solid which is pure glycerol. This data portrayed in Fig. 4 shows that the cooling time of 35 minutes has the lowest $K$ value which is 0.5 .

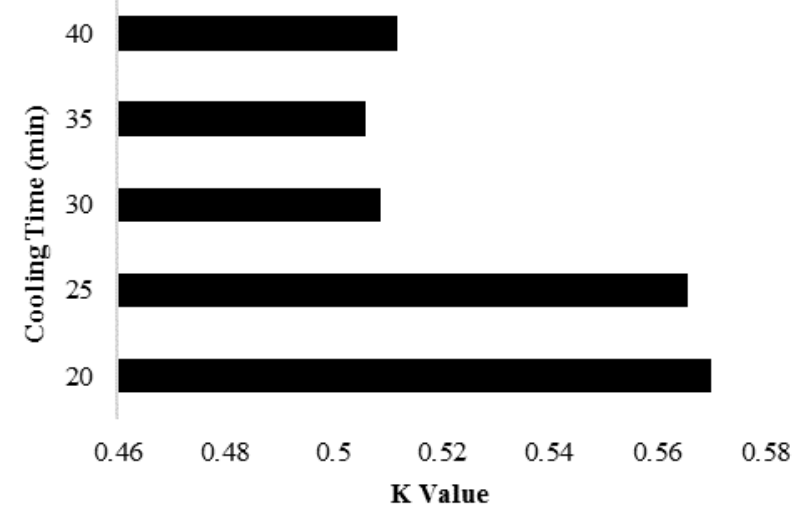

Fig. 4 Graph of $\mathrm{K}$ value for the effect of cooling time.

From Fig. 5, the value of efficiency increases from cooling time of 25 minutes until cooling time of 35 minutes. It shows that when the solution was stirred in a suitable time, the methyl ester was washed away from the solid front, leaving the solid in high purity (Chen et al., 1998). By having a suitable cooling time, a higher yield of pure methyl ester has been achieved. However, further increased in cooling time may cause several inclusions of the methyl ester into the solid growth where the diameter of the liquid path has been reduced. This has induced contamination of solute into the solid because of the saturation of the solute in the liquid phase. At this point, the purity of methyl ester shifted its trend where it starts to decrease from an increasing trend at 35 minutes. Therefore, the cooling time of 35 minutes has the highest concentration efficiency of $43.93 \%$ compared to the other cooling time throughout this experiment. The range of time 30 to 35 minutes was considered as the best cooling time since this range of time is not too long for the separation process to occur.

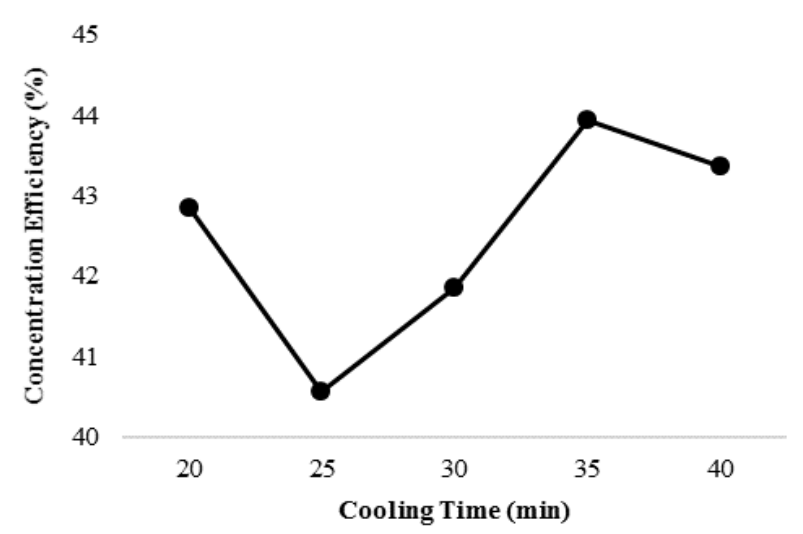

Fig. 5 Graph of concentration efficiency for the effect of cooling time.

\section{CONCLUSION}

In the present work, the fractional crystallization on separation and purification of methyl ester is applicable. The operating conditions that influence the separation of methyl ester from glycerol were tested using cylindrical crystallizer. To achieve highest effectiveness of fractional crystallization system and to obtain highest concentration efficiency, the fractional crystallization must operate in temperature range of $-10^{\circ} \mathrm{C}$ to $-12^{\circ} \mathrm{C}$ and cooling time range of 30 minutes to 35 minutes. It is important to operate the system with precision in order to form pure glycerol solid and recover the methyl 
ester in the solution. This study would be beneficial to measure its capability as a new technique for separating and purifying methyl ester.

\section{ACKNOWLEDGEMENT}

Authors would like to thank Universiti Teknologi PETRONAS for providing financial support through Short Term Internal Research Fund (STIRF) with Cost Centre 0153AA-F38, Sustainable Resources Mission Oriented Research for technical support and Centre for

Biofuel and Biochemical Research for facilities support.

\section{REFERENCES}

Aider, M., Ounis, W. B. 2012. Skim milk cryoconcentration as affected by the thawing mode: gravitational vs. microwave-assisted. Inter. J. of Food Sci. \& Tech. 47(1), 195-202.

Atadashi, I. M., Aroua, M. K., Abdul Aziz, A. R., Sulaiman, N. M. N. 2011 Membrane biodiesel production and refining technology: A critical review. Renew. and Sustain. Energy Rev. 15(9), 5051-5062.

Auleda, J. M., Raventós, M., Sánchez, J., Hernández, E. 2011. Estimation of the freezing point of concentrated fruit juices for application in freeze concentration. J. of Food Eng. 105(2), 289-294.

Berrios, M., Skelton, R. L. 2008. Comparison of purification methods for biodiesel. Chem. Eng. J. 144(3), 459-465.

Caretta, O., Courtot, F., Davies, T. 2006. Measurement of salt entrapment during the directional solidification of brine under forced mass convection. J. of Crystal Growth. 294(2), 151-155.

Chen, P., Chen, X. D., Free, K. W. 1998. Solute inclusion in ice formed from sucrose solutions on a sub-cooled surface - an experimental study. $J$. of Food Eng. 38(1), 1-13.

Fukui, K., Maeda, K., Kuramochi, H. 2013. Melt crystallization for refinement of triolein and palmitic acid mixture as a model waste oil for biodiesel fuel production. J. of Crystal Growth. 373, 102-105.

Hernández, E., Raventós, M., Auleda, J. M., Ibarz, A. 2010. Freeze concentration of must in a pilot plant falling film cryoconcentrator. Innovative Food Sci. \& Emerg. Tech. 11(1), 130-136.

Lemmer, S., Klomp, R., Ruemekorf, R., Scholz, R. 2001. Preconcentration of Wastewater through the Niro Freeze Concentration Process. Chem. Eng. \& Tech. 24(5), 485-488.

Mandri, Y., Rich, A., Mangin, D., Abderafi, S., Bebon, C., Semlali, N., et al. 2011. Parametric study of the sweating step in the seawater desalination process by indirect freezing. Des. 269(1-3), 142-147.

Matthews, J. S., Coggeshall, N. D. 1959. Concentration of Impurities from Organic Compounds by Progressive Freezing. Analytical Chem. 31(6), 1124-1125.

Miyawaki, O., Gunathilake, M., Omote, C., Koyanagi, T., Sasaki, T., Take, H. et al. 2016. Progressive freeze-concentration of apple juice and its application to produce a new type apple wine. J. of Food Eng. 171, 153158.

Miyawaki, O., Liu, L., Shirai, Y., Sakashita, S., Kagitani, K. 2005. Tubular ice system for scale-up of progressive freeze-concentration. J. of Food Eng. 69(1), 107-113.

Rane, M. V., Uphade, D. B. 2016. Energy Efficient Jaggery Making Using Freeze Pre-concentration of Sugarcane Juice. Energy Procedia. 90, 370381.

Rich, A., Mandri, Y., Mangin, D., Rivoire, A., Abderafi, S., Bebon, C., et al. 2012. Sea water desalination by dynamic layer melt crystallization: Parametric study of the freezing and sweating steps. J. of Crystal Growth. 342(1), 110-116.

Samsuri, S., Amran, N. A., Jusoh, M. 2015. Spiral finned crystallizer for progressive freeze concentration process. Chem. Eng. Res. and Des. 104, 280-286.

Samsuri, S., Amran, N. A., Yahya, N., Jusoh, M. 2016. Review on Progressive Freeze Concentration Designs. Chem. Eng. Commun. 203(3), 345-363.

Ulrich, J., Jones, M. J. 2006. Heat and mass transfer operations-crystallization. Chem. Eng. and Chem. Process Tech. 2.

Wintermantel, K., Wellinghoff, G. 2001. Layer Crystallization and Melt Solidification. In Crystallization Technology Handbook: CRC Press. 